\title{
Evolução Tardia após Intervenção Coronária Percutânea com Stents Farmacológicos em Pacientes Diabéticos do Registro DESIRE (Drug-Eluting Stents In the REal world)
}

\author{
Adriana Costa Moreira', Amanda G. M. R. Sousa ${ }^{1}$, José de Ribamar Costa Jr. ${ }^{1}$, Ricardo A. Costa', \\ Galo A. Maldonado ${ }^{1}$, Manuel N. Cano ${ }^{1}$, Ricardo Pavanello ${ }^{1}$, Otavio Berwanger ${ }^{1}$, \\ leda M. Liguori', Enilton S. T. Egito'1, Maria Helena Abib¹, J. Eduardo M. R. Sousa ${ }^{1}$
}

\section{RESUMO}

Fundamento: $O$ diabetes melito está relacionado à ocorrência de desfechos desfavoráveis após intervenções coronárias percutâneas. Os stents farmacológicos podem conferir melhor evolução tardia a esse subgrupo de pacientes. O objetivo deste estudo foi avaliar o desempenho desses instrumentais em pacientes diabéticos. Método: $O$ DESIRE é um registro unicêntrico, prospectivo, que incluiu 2.365 pacientes tratados consecutivamente com stents farmacológicos, entre maio de 2002 e janeiro de 2008. Para a presente análise foram excluídos pacientes com diagnóstico de infarto do miocárdio, aqueles com lesão tratada em ponte de safena e os com período de evolução inferior a 6 meses. Resultados: Assim, 1.705 pacientes foram divididos em dois grupos: não-diabéticos $(n=1.211$ pacientes, $71,1 \%$ ) e diabéticos $(n=494$ pacientes, 28,9\%), sendo $109(6,4 \%)$ pacientes em uso de insulina. Dentre os diabéticos, havia mais idosos $(64,8 \pm 9,8$ anos vs. $63,4 \pm$ $11,7$ anos; $p=0,025)$, mulheres $(28,9 \%$ vs. $22,5 \% ; p=0,005)$, obesos $(35,2 \%$ vs. $23,5 \%$; p < 0,001), hipertensos $(86,6 \%$ vs. $73,7 \%$; p < 0,001), multiarteriais $(63,4 \%$ vs. $54,4 \%$; $p=0,001)$, vasos finos $(2,70 \pm 0,51 \mathrm{~mm}$ vs. $2,75 \pm 0,42 \mathrm{~mm}$; p < 0,001) e calcificação na lesão tratada $(32,3 \%$ vs. $27,1 \% ; p=0,009)$. No seguimento clínico tardio $(2,2 \pm$ 1,1 anos), os eventos cardíacos combinados ocorreram mais freqüentemente no grupo dos pacientes diabéticos $(9,8 \%$ vs. $7,0 \%$; p $=0,048)$. A análise multivariada mostrou que o diabetes melito (odds ratio $[\mathrm{OR}]=1,45$; intervalo de confiança de 95\% [IC 95\%] = 1,0 a 2,1) e a presença de calcificação na lesão tratada $(O R=3,06$; IC 95\% = 1,47 a 6,34$)$ foram preditores independentes de eventos cardíacos maiores. Conclusão: O uso dos stents farmacológicos em diabéticos mostrou ser uma abordagem segura e eficiente. Os eventos cardíacos maiores combinados, embo-
SUMMARY

Long-Term Results after Percutaneous Coronary Intervention with Drug Eluting Stents in Diabetic Patients in the DESIRE (Drug-Eluting Stents In the REal world) Registry

Background: Diabetes mellitus is related to the occurrence of unfavorable outcomes after percutaneous coronary interventions. Drug-eluting stents can confer a better late evolution to this subgroup. The aim of this study was to assess the performance of these devices in diabetic patients. Methods: The DESIRE is a single-center, prospective registry that included 2,365 consecutive patients treated with drug-eluting stents between May 2002 and January 2008. For the present analysis, patients with a diagnosis of acute myocardial infarction, those with saphenous vein grafts lesions and those within 6 months of the index procedure, were excluded. Results: Therefore, 1,705 patients were divided into two groups: non-diabetics $(\mathrm{n}=1,211 \mathrm{P} / 71.1 \%)$ and diabetics ( $\mathrm{n}=494 \mathrm{P} / 28.9 \%)$, of which $109 \mathrm{P}(6.4 \%)$ were insulin-dependent. Among the diabetics there was a higher number of older people $(64.8$ \pm 9.8 years old vs. $63.4 \pm 11.7$ years old; $p=0.025)$, females (28.9 vs. $22.5 \% ; p=0.005)$, patients with obesity (35.2 vs. $23.5 \%$; $p<0.001$ ), hypertension (86.6 vs. $73.7 \%$; $\mathrm{p}<0.001)$, multi-vessel disease $(63.4$ vs. $54.4 \% ; p=0.001)$, small-vessel disease $(2.70 \pm 0.51 \mathrm{~mm}$ vs. $2.75 \pm 0.42 \mathrm{~mm}$; $p<0.001)$ and calcified lesions $(32.3$ vs. $27.1 \%$; $p=0.009)$. In the late clinical follow up $(2.2 \pm 1.1$ years $)$, the combined cardiac events occurred more frequently in the diabetic patients (9.8 vs. $7.0 \% ; p=0.048)$. The multivariate analysis showed that diabetes mellitus $(O R=1.45 ; 95 \%$ confidence interval 1.0 to 2.1 ) and the presence of a calcified lesion

\footnotetext{
1 Hospital do Coração da Associação do Sanatório Sírio - São Paulo, SP.

Correspondência: Adriana Costa Moreira. Rua Capitão Macedo, 171 - Apto. 73 - Vila Clementino - São Paulo, SP - CEP 04021-020 E-mail: moreira.adriana@uol.com.br

Recebido em: 8/1/2008 - Aceito em: 22/5/2008
} 
Moreira AC, et al. Evolução Tardia após Intervenção Coronária Percutânea com Stents Farmacológicos em Pacientes Diabéticos do Registro DESIRE (Drug-Eluting Stents In the REal world). Rev Bras Cardiol Invas. 2008;16(2):185-192.

ra em taxas muito baixas, ocorreram mais freqüentemente no grupo dos diabéticos.

DESCRITORES: Diabetes melito. Endoprótese, efeitos adversos. Angioplastia transluminal percutânea coronária. Resultado de tratamento.
$(\mathrm{OR}=3.06 ; 95 \% \mathrm{Cl} 1.47$ to 6.34$)$ were independent predictors of major adverse cardiac events. Conclusion: The use of drug-eluting stents in diabetics showed to be a safe and efficient approach. The major adverse cardiac events, although in very low rates, occurred more frequently in the diabetics.

DESCRIPTORS: Diabetes mellitus. Stents, adverse effects. Angioplasty, transluminal, percutaneous coronary. Treatment outcome.
0 diabetes melito é tradicionalmente relacionado à ocorrência de desfechos clínicos desfavoráveis após intervenções coronárias percutâneas'. O estilo de vida atual e o envelhecimento populacional constituem as principais causas do crescente aumento da incidência dessa enfermidade.

Vários estudos randomizados com populações selecionadas tratadas com stents farmacológicos demonstraram ampliação dos benefícios clínicos da intervenção coronária percutânea para diversos subgrupos clínicos e angiográficos considerados mais complexos, inclusive os diabéticos. A partir desses resultados, houve grande aumento da utilização desses dispositivos no "mundo-real".

O objetivo deste estudo foi avaliar a segurança e a efetividade dos stents farmacológicos implantados em pacientes diabéticos da rotina prática diária, comparando-os aos não-diabéticos.

\section{MÉTODO}

\section{Casuística e desenho do estudo}

Desde maio de 2002, quando os stents farmacológicos tiveram sua utilização liberada pelos órgãos regulamentadores de nosso País, todos os pacientes tratados exclusivamente com esses instrumentais no Hospital do Coração da Associação do Sanatório Sírio, em São Paulo, foram incluídos no Registro DESIRE (Drug-Eluting Stent In the REal world) ${ }^{2}$. Trata-se de um estudo prospectivo, unicêntrico, não-randomizado de casos consecutivos. Um banco de dados especialmente dedicado ao Registro DESIRE foi desenvolvido, no qual constam dados pormenorizados das características clínicas e angiográficas e informações técnicas da intervenção de cada um dos pacientes registrados. Os dados evolutivos hospitalares e os obtidos nos períodos definidos (1, 6, 12 meses e anualmente a partir de então) após o procedimento-índice foram também registrados. As taxas de seguimento foram calculadas, considerando-se o número de pacientes acompanhados em relação ao número de casos elegíveis que tivessem atingido aquela etapa predeterminada do acompanhamento.

No Registro DESIRE foram incluídos todos os pacientes com indicação de intervenção coronária per- cutânea, independentemente do quadro clínico ou angiográfico, excluindo-se apenas aqueles pacientes com contra-indicação para o uso prolongado dos agentes antiplaquetários definidos no protocolo medicamentoso. Para a presente análise foram excluídos também aqueles cuja apresentação clínica inicial era o infarto do miocárdio, assim como os pacientes com pontes de safena tratadas e os que não haviam completado 6 meses desde a intervenção. A amostra foi dividida em dois grupos, de acordo com a presença ou não de diabetes melito.

\section{Terapêutica antitrombótica}

O protocolo antitrombótico consistiu na administração de dois agentes antiplaquetários: o ácido acetilsalicílico (AAS) e um tienopiridínico. O AAS (100200 mg diários) era iniciado pelo menos 24 horas antes do procedimento e mantido indefinidamente. $\mathrm{O}$ clopidogrel (dose de ataque de 300-600 mg) era administrado 24 horas antes da intervenção, seguido da dose diária de $75 \mathrm{mg}$, e a ticlopidina (250 mg a cada 12 horas) era iniciada pelo menos 48 horas antes do procedimento. Até dezembro de 2006, a recomendação consistia no uso do tienopiridínico durante 3 a 6 meses após implante dos stents Cypher ${ }^{\circledR}$ e Taxus $^{\mathrm{TM}}$, respectivamente. Desde 2007, a duração da terapêutica antiplaquetária com tienopiridínico foi prolongada para um ano, independentemente do stent farmacológico implantado. Durante o procedimento, a heparina foi administrada na dose de $70 \mathrm{UI} / \mathrm{kg}$ a $100 \mathrm{UI} / \mathrm{kg}$ e doses adicionais para manter o tempo de coagulação ativado $>250$ segundos. A decisão sobre a utilização dos inibidores da glicoproteína Ilb/Illa ficou a critério do operador.

\section{Intervenção coronária percutânea}

O implante do stent farmacológico seguiu a técnica contemporânea estabelecida. Sempre que necessária, a pré-dilatação deveria ser realizada com balões curtos insuflados a baixas pressões, visando a evitar injúria nos segmentos adjacentes à lesão. O stent deveria ser implantado de forma a garantir a completa cobertura da lesão e, quando necessário, mais de um stent poderia ser realizado com a sobreposição das bordas dos mesmos. Após o implante de stent, a pós-dilata- 
Moreira AC, et al. Evolução Tardia após Intervenção Coronária Percutânea com Stents Farmacológicos em Pacientes Diabéticos do Registro DESIRE (Drug-Eluting Stents In the REal world). Rev Bras Cardiol Invas. 2008;16(2):185-192.

ção, quando indicada, deveria ser realizada com balões com extensão menor que a do stent implantado, com o cuidado de não ultrapassar suas bordas, evitando mais uma vez a injúria nos segmentos não cobertos pela prótese. Utilizamos os seguintes stents farmacológicos: stent Cypher ${ }^{\circledR}$ (Cordis, Johnson \& Johnson), com eluição de sirolimus; stent Taxus ${ }^{\mathrm{TM}}$ (Boston Scientific Corporation), com eluição de paclitaxel; e stent Endeavor $^{\mathrm{TM}}$ (Medtronic Corporation), com eluição de zotarolimus. Esses stents estavam disponíveis em variações de extensão de $8 \mathrm{~mm}$ a $33 \mathrm{~mm}$ e diâmetros de 2,25 mm a 3,5 mm para o stent Cypher ${ }^{\circledR}$, extensões de $8 \mathrm{~mm}$ a $32 \mathrm{~mm}$ e diâmetros de $2,25 \mathrm{~mm}$ a $4,5 \mathrm{~mm}$ para o stent Taxus ${ }^{\text {TM }}$, e extensões de $8 \mathrm{~mm}$ a $30 \mathrm{~mm}$ e diâmetros de 2,25 mm a 4,0 mm para o stent Endeavor $^{\mathrm{TM}}$. A escolha do tipo de stent farmacológico ficou a critério de cada operador.

A angiografia coronária quantitativa foi realizada imediatamente antes e após o implante do stent, por técnico treinado e experiente. A análise do segmento tratado compreendeu a área coberta pelo stent e as bordas $(5 \mathrm{~mm})$ proximal e distal. As mensurações angiográficas incluíram as referências proximal, distal e interpolada, o diâmetro mínimo da luz (DML), a porcentagem de obstrução e a extensão da lesão. Além disso, o ganho imediato foi avaliado a partir do cálculo da diferença entre o DML aferido imediatamente após e antes do implante do stent.

Durante a hospitalização, todos os pacientes foram submetidos a avaliação com eletrocardiograma (ECG) e marcadores bioquímicos (CK-MB), em três momentos: antes e imediatamente após a intervenção e na alta hospitalar.

\section{Objetivos do estudo e definições}

O desfecho primário deste estudo foi a avaliação da ocorrência de eventos cardíacos maiores no seguimento clínico tardio (> 6 meses). Os desfechos secundários incluíram as taxas de revascularização da lesão-alvo (RLA) e trombose do stent em todo o período do seguimento.

Os eventos cardíacos maiores foram definidos como óbito cardíaco, infarto do miocárdio e RLA. Os óbitos foram classificados como cardíacos e não-cardíacos. Óbitos de causas indeterminadas foram relatados como cardíacos. Os infartos do miocárdio foram definidos como o aparecimento de novas ondas $Q$ em duas derivações contíguas do ECG e/ou a elevação da CK$\mathrm{MB}>3$ vezes o limite superior do normal. Todas as reintervenções, percutâneas ou cirúrgicas, resultantes da recorrência da obstrução (> 50\%) dentro do stent implantado no procedimento-índice ou no segmento vascular, que incluía os $5 \mathrm{~mm}$ proximais e os $5 \mathrm{~mm}$ distais ao stent, foram classificadas como RLA.

A classificação proposta pelo Academic Research Consortium foi utilizada para a definição da trombose do stent em definitiva (confirmação angiográfica ou anatomopatológica), provável (morte súbita $\leq 30$ dias pós-stents farmacológicos ou infarto relacionado à região da artéria tratada, mesmo sem confirmação angiográfica) e possível (morte súbita > 30 dias pós-stents farmacológicos). Quanto à distribuição temporal, as tromboses protéticas ainda foram divididas como: agudas e subagudas ( $\leq 30$ dias), tardias (31 dias a 360 dias) e muito tardias (> 360 dias).

O sucesso angiográfico foi definido como o achado de lesão residual $<20 \%$ associado à presença de fluxo coronário TIMI 3 na ausência de trombos e/ou dissecções. O sucesso do procedimento foi considerado como o sucesso angiográfico na ausência de eventos cardíacos maiores.

A função ventricular esquerda foi avaliada pelo cálculo da fração de ejeção (\% FE) e classificada como: a) normal (\% FE $\geq 55 \%$ ); b) disfunção discreta (\% FE $\geq 40 \%<55 \%$ ); c) disfunção moderada (\% FE $\geq 30 \%$ $<40 \%$ ); e d) disfunção significativa (\% FE < 30\%).

A insuficiência renal crônica foi definida pela presença de valores séricos da creatinina $\geq 1,5 \mathrm{mg} / \mathrm{dl}$ e pela taxa de filtração glomerular (clearance) $<60 \mathrm{ml} /$ $\min / 1,73 \mathrm{~m}^{2}$.

Definimos como indicações off-label o grupo de pacientes que receberam stents farmacológicos e não preenchiam os critérios padrão do Food and Drug Administration (FDA), ou seja, lesões reestenóticas, extensão da lesão $>30 \mathrm{~mm}$, diâmetro de referência do vaso tratado menor que $2,5 \mathrm{~mm}$ ou maior que $3,75 \mathrm{~mm}$, lesões em tronco da artéria coronária esquerda nãoprotegido, bifurcações e oclusões crônicas. O infarto do miocárdio como apresentação clínica e o tratamento de pontes de safena são usualmente considerados condições off-label; entretanto, não foram incluídos nesta descrição por consistirem critérios de exclusão da presente investigação.

\section{Análise estatística}

Devido à alta variabilidade dos dados, as variáveis contínuas foram apresentadas como mediana $1^{\underline{0}}$ e $3^{\text {o }}$ quartis e comparadas em relação aos grupos pelo teste não-paramétrico de Kruskall-Wallis (quando se avaliaram 3 grupos de diabetes) ou pelo teste de Mann-Whitney (comparação entre diabéticos e não-diabéticos).

As variáveis categóricas foram apresentadas em números e porcentuais e comparadas entre os grupos de diabetes pelo teste do qui-quadrado ou exato de Fisher, quando apropriado. A incidência acumulada de eventos cardíacos foi estimada de acordo com o método de Kaplan-Meier e as diferenças entre as curvas de sobrevivência livre de eventos foram comparadas pelo teste de Breslow, em decorrência da não proporcionalidade das curvas de sobrevida. Valores de $p<0,05$ foram considerados significantes. 
Moreira AC, et al. Evolução Tardia após Intervenção Coronária Percutânea com Stents Farmacológicos em Pacientes Diabéticos do Registro DESIRE (Drug-Eluting Stents In the REal world). Rev Bras Cardiol Invas. 2008;16(2):185-192.

Após a identificação das características associadas ao diabetes melito, um modelo de regressão logística foi considerado, estratificando os pacientes em diabéticos e não-diabéticos. Todas as variáveis que tiveram valor de $p<0,1$ na análise bruta foram consideradas possíveis preditores de eventos no registro DESIRE.

A análise estatística foi realizada com a utilização do software SPSS 16.0 (SPSS Inc., Chicago, Illinois, Estados Unidos).

\section{RESULTADOS}

Entre maio de 2002 e janeiro de 2008, 2.365 pacientes foram consecutivamente tratados apenas com stents farmacológicos e incluídos no Registro DESIRE. Destes, 660 indivíduos apresentavam lesão-alvo em pontes de safena, diagnóstico clínico de infarto do miocárdio e/ou tinham menos de 6 meses desde o procedimento inicial com stents farmacológicos, sendo, dessa forma, excluídos desta investigação. Os 1.705 pacientes restantes foram divididos em dois grupos: não-diabéticos ( $\mathrm{n}=1.211$ pacientes, $71,1 \%$ ) e diabéticos ( $n=494$ pacientes, 28,9\%), estando $109(6,4 \%)$ desses pacientes em uso de insulina.

As características clínicas e angiográficas dos dois grupos estão descritas na Tabela 1. No grupo dos pacientes diabéticos, a média das idades foi mais elevada $(64,8 \pm 9,8$ anos vs. 63,4 \pm 11,7 anos; $p=0,025)$. Além disso, havia maior proporção de mulheres $(28,9 \%$ vs. $22,5 \%$; p = 0,005), obesos $(35,2 \%$ vs. $23,5 \%$; $\mathrm{p}<0,001)$, portadores de hipertensão arterial $(86,6 \%$ vs. $73,7 \%$; $p<0,001)$, histórico de acidente vascular cerebral $(3,4 \%$ vs. $1,4 \% ; p=0,006)$ e doença arterial periférica $(7,9 \%$ vs. 3,5\%; $p<0,001)$.

Não houve diferenças significativas entre os grupos quando comparados em relação à apresentação clínica inicial, sendo a angina instável o quadro clínico apresentado por cerca de um terço dos pacientes nos dois grupos $(29,2 \%$ vs. $33,4 \% ; p=0,11)$.

O comprometimento multiarterial foi mais freqüente entre os diabéticos $(63,4 \%$ vs. 54,4\%; p = 0,001), assim como a presença de calcificação moderada/intensa $(32,3 \%$ vs. $27,1 \% ; p=0,009)$.

O uso do stent Cypher $^{\circledR}$ foi predominante nesse registro, sem diferença entre os grupos $(87,2 \%$ nos diabéticos e $85,3 \%$ nos pacientes não-diabéticos).

A Tabela 2 apresenta os aspectos quantitativos dos vasos e das lesões tratadas e os dados dos procedimentos. Podemos observar que, nos pacientes diabéticos, o diâmetro de referência dos vasos tratados foi menor que nos não-diabéticos $(2,70 \pm 0,51 \mathrm{~mm}$ vs. 2,75 $\pm 0,42 \mathrm{~mm}$; $p<0,001)$ e a pressão de liberação do stent foi inferior àquela do grupo dos pacientes não-diabéticos (14,43 \pm $2,75$ atm vs. $14,74 \pm 2,83$ atm; $p=0,015)$.

No seguimento clínico tardio (2,5 \pm 1,4 anos), os eventos cardíacos acumulados analisados de forma combinada ocorreram com maior freqüência entre os pacientes diabéticos $(9,8 \%$ vs. $7,0 \% ; p=0,048)$. Os eventos isolados, como óbito cardíaco, infarto do miocárdio e RLA, ocorreram de forma similar em ambos os grupos (Tabela 3). A sobrevida livre de eventos foi calculada nos dois grupos: não-diabéticos e diabéticos (Figura 1). Houve comportamento semelhante nos dois grupos analisados, inclusive com relação à ocorrência de trombose do stent (Tabela 4).

$\mathrm{Na}$ amostra analisada, a análise multivariada demonstrou que a presença de diabetes melito foi um preditor independente de eventos cardíacos maiores (odds ratio $[\mathrm{OR}]=1,45$; intervalo de confiança de $95 \%$ [IC 95\%] = 1,0 a 2,1). Além disso, nos pacientes diabéticos, a presença de calcificação moderada/intensa na lesão tratada também esteve relacionada a maior ocorrência de eventos cardíacos maiores $(\mathrm{OR}=3,06 ;$ IC $95 \%=1,47$ a 6,34$)$.

\section{DISCUSSÃO}

Nesta experiência, os bons resultados do seguimento tardio de pacientes da rotina diária submetidos a intervenção coronária percutânea com stents farmacológicos demonstram elevado perfil de segurança e eficiência desses instrumentais, mesmo em pacientes diabéticos. Essa abordagem terapêutica pode ser considerada, portanto, uma alternativa para o tratamento da doença coronária obstrutiva nesse complexo subgrupo de pacientes.

As análises dos subgrupos dos pacientes diabéticos dos primeiros estudos randomizados da era dos stents farmacológicos comparando-os aos stents nãofarmacológicos corroboram, na sua maioria, os presentes achados ${ }^{3-6}$.

Sabaté et al. ${ }^{7}$ analisaram 160 pacientes diabéticos randomizados para o uso de stents não-farmacológicos ou com eluição de sirolimus. No seguimento de 9 meses, as taxas de sobrevivência livre de eventos cardíacos maiores foram de $63,7 \%$ e $88,7 \%$, respectivamente $(p=0,005)$. Houve redução significante na necessidade de novas revascularizações após o implante dos stents farmacológicos $(7,5 \%$ vs. 31,3\%; $\mathrm{p}<0,0001)$. Os pacientes diabéticos em uso de insulina obtiveram o mesmo benefício na redução da reestenose quando comparados àqueles em uso de hipoglicemiantes orais. Além disso, o uso desses instrumentais foi considerado seguro pela não ocorrência de tromboses tardias.

Diante dos resultados iniciais, a ampliação do uso dos stents farmacológicos para casos mais complexos foi inevitável, incluindo comumente aqueles pacientes com características clínicas e/ou angiográficas consideradas critérios de exclusão nos estudos randomizados.

Diante dos ainda recentes questionamentos sobre a segurança dos stents farmacológicos, os dados pro- 
Moreira AC, et al. Evolução Tardia após Intervenção Coronária Percutânea com Stents Farmacológicos em Pacientes Diabéticos do Registro DESIRE (Drug-Eluting Stents In the REal world). Rev Bras Cardiol Invas. 2008;16(2):185-192.

TABELA 1

Características clínicas e angiográficas

\begin{tabular}{|c|c|c|c|}
\hline & $\begin{array}{c}\text { Não-diabéticos } \\
(\mathrm{n}=\mathbf{1 . 2 1 1} \text { pacientes })\end{array}$ & $\begin{array}{c}\text { Diabéticos } \\
(\mathrm{n}=494 \text { pacientes })\end{array}$ & $\mathbf{p}$ \\
\hline Idade (média \pm DP, anos) & $63,4 \pm 11,7$ & $64,8 \pm 9,8$ & 0,025 \\
\hline Sexo feminino, n (\%) & $272(22,5)$ & $143(28,9)$ & 0,005 \\
\hline Hipertensão arterial, n (\%) & $893(73,7)$ & $428(86,6)$ & $<0,001$ \\
\hline Hipercolesterolemia, n (\%) & $753(62,2)$ & $305(61,7)$ & 0,53 \\
\hline Tabagismo, n (\%) & $110(9,1)$ & $36(7,3)$ & 0,33 \\
\hline Obesidade, n (\%) & $278(23,5)$ & $166(35,2)$ & $<0,001$ \\
\hline Insuficiência renal crônica, n (\%) & $107(9,7)$ & $52(11,9)$ & 0,20 \\
\hline Acidente vascular cerebral, n (\%) & $17(1,4)$ & $17(3,4)$ & 0,006 \\
\hline Doença arterial periférica, n (\%) & $42(3,5)$ & $39(7,9)$ & $<0,001$ \\
\hline Histórico familiar de doença coronária, n (\%) & $563(47,4)$ & $207(41,9)$ & 0,041 \\
\hline Infarto prévio, n (\%) & $234(19,3)$ & $100(20,2)$ & 0,66 \\
\hline Intervenção coronária percutânea prévia, n (\%) & $312(25,8)$ & $123(24,9)$ & 0,71 \\
\hline Revascularização miocárdica cirúrgica, n (\%) & $275(22,7)$ & $126(25,5)$ & 0,21 \\
\hline Apresentação clínica, n (\%) & & & 0,11 \\
\hline Isquemia silenciosa & $440(36,3)$ & $182(36,8)$ & \\
\hline Angina estável & $417(34,4)$ & $147(29,8)$ & \\
\hline Angina instável & $354(29,2)$ & $165(33,4)$ & \\
\hline Extensão da doença coronária, n (\%) & & & 0,001 \\
\hline 1 vaso & $551(45,6)$ & $180(36,6)$ & \\
\hline 2 vasos & $396(32,8)$ & $168(34,1)$ & \\
\hline 3 vasos/TCE & $261(21,6)$ & $144(29,3)$ & \\
\hline Função ventricular esquerda & & & 0,19 \\
\hline Normal & $168(77,4)$ & $58(68,2)$ & \\
\hline Disfunção discreta & $29(13,4)$ & $16(18,8)$ & \\
\hline Disfunção moderada & $18(8,3)$ & $8(9,4)$ & \\
\hline Disfunção importante & $2(0,9)$ & $3(3,5)$ & \\
\hline Uso de inibidor da glicoproteína llb/Illa & $13(1,1)$ & $6(1,3)$ & 0,80 \\
\hline Indicações off-label & $862(71,2)$ & $368(74,5)$ & 0,16 \\
\hline Procedimento multiarterial & $300(24,8)$ & $137(27,7)$ & 0,20 \\
\hline Número de vasos tratados & 1.476 & 653 & N/A \\
\hline Número de vasos tratados por paciente & 1,27 & 1,32 & 0,14 \\
\hline Vaso tratado & & & 0,58 \\
\hline Artéria descendente anterior & $652(44,2)$ & $269(43,4)$ & \\
\hline Artéria circunflexa & $426(28,9)$ & $181(29,2)$ & \\
\hline Artéria coronária direita & $384(26,0)$ & $162(26,1)$ & \\
\hline Tronco da artéria coronária esquerda & $14(1,0)$ & $8(1,3)$ & \\
\hline Número de lesões tratadas & 1.634 & 708 & N/A \\
\hline Número de lesões tratadas por paciente & 1,35 & 1,43 & 0,06 \\
\hline Lesões reestenóticas & $76(4,6)$ & $33(4,4)$ & 0,77 \\
\hline Tipo de lesão tratada*, n (\%) & & & 0,20 \\
\hline A & $54(5,2)$ & $13(2,6)$ & \\
\hline B1 & $334(32,1)$ & $148(30,0)$ & \\
\hline B2 & $401(38,5)$ & $209(42,4)$ & \\
\hline $\mathrm{C}$ & $252(24,2)$ & $123(25,0)$ & \\
\hline Bifurcação (ramo lateral > 2 mm) & $140(7,8)$ & $35(4,6)$ & 0,004 \\
\hline Calcificação moderada/intensa & $488(27,1)$ & $245(32,3)$ & 0,009 \\
\hline
\end{tabular}

*Classificação da American Heart Association e do American College of Cardiology (AHA/ACC). DP = desvio padrão; $\mathrm{n}=$ número de pacientes; TCE $=$ tronco coronário esquerdo. 
TABELA 2

Angiografia coronária quantitativa e dados dos procedimentos

\begin{tabular}{|c|c|c|c|}
\hline & $\begin{array}{c}\text { Não-diabéticos } \\
\text { (n = 1.634 lesões) }\end{array}$ & $\begin{array}{c}\text { Diabéticos } \\
\text { (n = } 708 \text { lesões) }\end{array}$ & $\mathbf{p}$ \\
\hline \multicolumn{4}{|l|}{ Angiografia coronária quantitativa } \\
\hline \multicolumn{4}{|l|}{ Pré-procedimento } \\
\hline Diâmetro de referência (média \pm DP), mm & $2,75 \pm 0,42$ & $2,70 \pm 0,51$ & $<0,001$ \\
\hline Extensão das lesões (média \pm DP), mm & $17,63 \pm 10,23$ & $17,67 \pm 9,20$ & 0,33 \\
\hline$\%$ estenose & $66,70 \pm 12,82$ & $66,50 \pm 11,01$ & 0,66 \\
\hline Diâmetro mínimo da luz (mm) & $1,13 \pm 2,35$ & $0,99 \pm 0,99$ & 0,08 \\
\hline \multicolumn{4}{|l|}{ Pós-procedimento } \\
\hline Lesão residual (\%) & $4,92 \pm 3,65$ & $4,98 \pm 3,97$ & 0,15 \\
\hline Diâmetro mínimo da luz (mm) & $2,89 \pm 1,10$ & $2,80 \pm 0,91$ & 0,04 \\
\hline Ganho imediato (média/mm) & 1,76 & 1,81 & 0,7 \\
\hline Implante direto, n (\%) & $873(48,6)$ & $360(47,4)$ & 0,60 \\
\hline Número de stents implantados & 1.798 & 759 & \\
\hline Número de stents por paciente & 1,48 & 1,53 & 0,02 \\
\hline Diâmetro dos stents implantados (média \pm DP), mm & $2,87 \pm 0,37$ & $2,82 \pm 0,38$ & 0,1 \\
\hline Extensão dos stents implantados (média \pm DP), mm & $19,06 \pm 5,68$ & $19,53 \pm 6,25$ & 0,33 \\
\hline Relação: extensão dos stents/extensão das lesões & 1,08 & 1,10 & 0,3 \\
\hline Pressão de liberação (média \pm DP), atm & $14,74 \pm 2,83$ & $14,43 \pm 2,75$ & 0,015 \\
\hline Sobreposição de stents & $195(10,8)$ & $55(7,2)$ & 0,005 \\
\hline Sucesso angiográfico & $99,1 \%$ & $98,9 \%$ & 0,9 \\
\hline Pós-dilatação, n (\%) & $788(43,8)$ & $347(45,7)$ & 0,37 \\
\hline
\end{tabular}

TABELA 3

Eventos cardíacos no seguimento clínico tardio

\begin{tabular}{lccr}
\hline Eventos acumulados, $\mathbf{n}(\mathbf{\%})$ & $\begin{array}{c}\text { Não-diabéticos } \\
(\mathbf{n}=\mathbf{1 . 2 0 5} \text { pacientes) }\end{array}$ & $\begin{array}{c}\text { Diabéticos } \\
\text { (n = 489 pacientes) }\end{array}$ & $\mathbf{p}$ \\
\hline Óbito cardíaco & $24(2,0)$ & $15(3,1)$ & 0,18 \\
Infarto do miocárdio & $32(2,7)$ & $18(3,7)$ & 0,25 \\
Revascularização da lesão-alvo & $30(2,5)$ & $18(3,7)$ & 0,17 \\
Eventos cardíacos maiores combinados & $84(7,0)$ & $48(9,8)$ & 0,048 \\
\hline
\end{tabular}

$\mathrm{n}=$ número de pacientes.

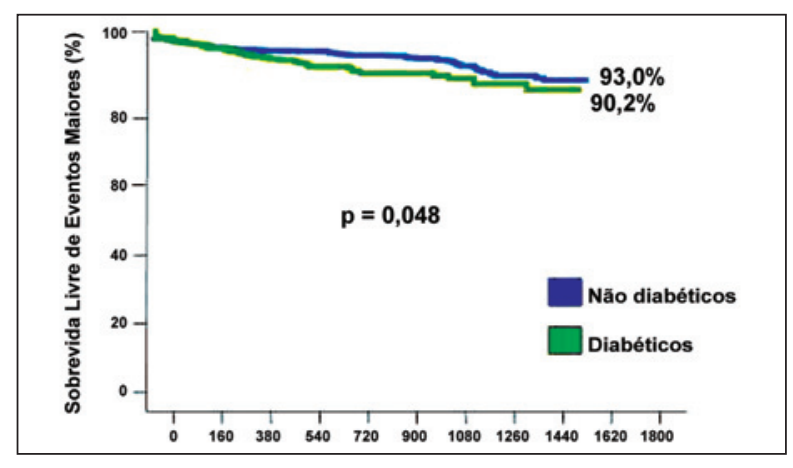

Figura 1 - Curvas de sobrevida livre de eventos cardíacos maiores: diabéticos versus não-diabéticos. venientes da experiência global no chamado "mundoreal" estão sendo cada vez mais relevantes, uma vez que incluem um número considerável de pacientes com indicações consideradas off-label. Os registros têm trazido contribuições valiosas nesse sentido ${ }^{8,9}$.

Em recente publicação, Mulukutla et al. ${ }^{10}$ descreveram os resultados do seguimento clínico de um ano de 2.566 pacientes diabéticos, dividindo-os de acordo com a utilização ou não de insulina e com o tipo de stent empregado, não-farmacológico ou farmacológico. O uso dos stents farmacológicos quando comparado ao dos não-farmacológicos esteve associado a menor risco de novas revascularizações, tanto no gru- 
Moreira AC, et al. Evolução Tardia após Intervenção Coronária Percutânea com Stents Farmacológicos em Pacientes Diabéticos do Registro DESIRE (Drug-Eluting Stents In the REal world). Rev Bras Cardiol Invas. 2008;16(2):185-192.

TABELA 4

Classificação das tromboses protéticas (Academic Research Consortium)

\begin{tabular}{lccc}
\hline Trombose do stent, $\%(\mathbf{n})$ & Não-diabéticos $(\mathbf{n}=\mathbf{1 . 2 1 1}$ pacientes $)$ & Diabéticos $(\mathbf{n}=\mathbf{4 9 4}$ pacientes $)$ & $\mathbf{p}$ \\
\hline Total & $1,2(13)$ & $1,8(9)$ & 0,21 \\
Definitiva & $(9)$ & $(4)$ & \\
Provável & $(0)$ & $(1)$ & \\
Possível & $(4)$ & $(4)$ & \\
Aguda & $(0)$ & $(0)$ \\
Subaguda & $(1)$ & $(3)$ \\
Tardia & $(8)$ & $(2)$ & \\
Muito tardia & $(4)$ & $(4)$ & \\
\hline
\end{tabular}

po de pacientes em uso de insulina (razão de risco ajustada $[R R]=0,63, \mathrm{IC} 95 \%=0,44$ a 0,90$)$ como naqueles que não a utilizavam ( $R R=0,59$, IC $95 \%=0,45$ a 0,76$)$. Com relação à segurança na população geral de diabéticos, os stents farmacológicos estiveram associados à redução das taxas de óbito ou infarto do miocárdio $(R R=0,75, I C \quad 95 \%=0,58$ a 0,96). Entretanto, esse benefício foi restrito ao subgrupo de pacientes sem uso de insulina ( $R R=0,57$, IC $95 \%=0,41$ a 0,81 ). Nos pacientes diabéticos tratados com insulina, não houve diferenças na ocorrência de óbito ou infarto do miocárdio entre os subgrupos tratados com stents farmacológicos ou não-farmacológicos $(R R=0,95, I C$ $95 \%=0,65$ a 1,39). Nossos resultados também apontam para a equalização dos resultados entre pacientes diabéticos e não-diabéticos, mesmo quando analisamos aqueles em uso de insulina, cuja evolução clínica foi semelhante à dos demais, diabéticos e não-diabéticos, com taxas de eventos cardíacos superiores a 9,4\%, $7,0 \%$ e $11,2 \%$, respectivamente $(p=0,12)$.

Por outro lado, os dados de literatura do stent Taxus $^{\text {TM }}$ referente aos pacientes diabéticos restringemse à análise dos estudos randomizados. Os resultados tardios (quatro anos) dos estudos da série TAXUS (I, II, IV, V e VI) demonstraram não haver diferenças entre os stents com eluição de paclitaxel (Taxus ${ }^{\text {TM) }}$ e os stents convencionais em pacientes diabéticos, no que se refere a taxas de óbito, infarto do miocárdio e trombose do stent. Houve, no entanto, significativa redução das taxas de RLA nos pacientes tratados com os stents farmacológicos $(12,4 \% \text { vs. } 24,7 \% ; p<0,0001)^{11}$.

Por fim, as taxas de trombose protética encontradas nos dois grupos, não-diabéticos e diabéticos, foram consideradas baixas, uma vez que estamos diante de uma população de risco mais elevado. A recomendação para o uso prolongado da terapêutica antiplaquetária, assim como o grande esforço em manter a adesão dos pacientes à terapêutica instituída, devem ter contribuído para esse fato. Observamos ainda que as taxas de trombose do stent dos dois grupos analisados neste estudo não atingiram diferença estatisticamente significativa $(1,2 \%$ vs. $1,8 \% ; p=0,21)$.

\section{Limitações}

O presente estudo é um registro unicêntrico e não-randomizado. Esta análise não se presta à comparação dos diferentes stents farmacológicos, uma vez que o stent $\mathrm{Cypher}^{\circledR}$ foi predominantemente implantado no Registro DESIRE. A ausência de reestudo angiográfico, embora espelhe a realidade da prática clínica rotineira, impossibilita análises referentes ao desempenho desses instrumentais em reduzir a hiperplasia intimal e a reestenose binária.

\section{CONCLUSÕES}

No Registro DESIRE, o seguimento clínico tardio demonstrou que a intervenção coronária percutânea com implante dos stents farmacológicos consiste numa abordagem segura e eficiente para o tratamento da doença arterial coronária obstrutiva em pacientes diabéticos. Quando analisados de forma combinada, os eventos cardíacos maiores, embora em taxas muito baixas, ocorreram mais freqüentemente no grupo de diabéticos. Esses dados apontam para a necessidade de aperfeiçoamento técnico e dos instrumentais, mas também do controle intenso das repercussões sistêmicas da doença.

\section{REFERÊNCIAS BIBLIOGRÁFICAS}

1. Abizaid A, Kornowski R, Mintz GS, Hong MK, Abizaid AS, Mehran R, et al. The influence of diabetes mellitus on acute and late clinical outcomes following coronary stent implantation. J Am Coll Cardiol. 1998;32(3):584-9.

2. Sousa AGMR, Costa Junior JR, Moreira A, Costa RA, Cano MN, Andrade GAM, et al. Evolução clínica tardia dos stents farmacológicos: segurança e eficácia até cinco anos do registro DESIRE. Rev Bras Cardiol Invas. 2007;15(3):221-7.

3. Abizaid A, Costa MA, Blanchard D, Albertal M, Eltchaninoff $\mathrm{H}$, Guagliumi G, et al. Sirolimus-eluting stents inhibit neointimal hyperplasia in diabetic patients: insights from the RAVEL trial. Ravel Investigators. Eur Heart J. 2004;25(2):107-12.

4. Moussa I, Leon MB, Baim DS, O'Neill WW, Popma JJ, Buchbinder $M$, et al. Impact of sirolimus-eluting stents on outcome in diabetic patients: a SIRIUS (SIRolImUS-coated Bx Velocity balloon-expandable stent in the treatment of patients with de novo coronary artery lesions) substudy. Circulation. 2004;109(19):2273-8. 
5. Hermiller JB, Raizner A, Cannon L, Gurbel PA, Kutcher MA, Wong SC, et al. Outcomes with the polymer-based paclitaxeleluting TAXUS stent in patients with diabetes mellitus: the TAXUS-IV trial. TAXUS-IV Investigators. J Am Coll Cardiol. 2005;45(8):1172-9.

6. Spaulding C, Daemen J, Boersma E, Cutlip DE, Serruys PW. A pooled analysis of data comparing sirolimus-eluting stents with bare-metal stents. N Engl J Med. 2007;356(10):989-97.

7. Sabaté $M$, Jiménez-Quevedo P, Angiolillo DJ, Gómez-Hospital JA, Alfonso F, Hernandez-Antolin R, et al. Randomized comparison of sirolimus-eluting stent versus standard stent for percutaneous coronary revascularization in diabetic patients. The Diabetes and Sirolimus-Eluting Stent (DIABETES) trial. DIABETES Investigators. Circulation. 2005;112(14):2175-83.

8. Kuchulakanti PK, Torguson R, Canos D, Rha SW, Chu WW, Clavijo L, et al. Impact of treatment of coronary artery disease with sirolimus-eluting stents on outcomes of diabetic and nondiabetic patients. Am J Cardiol. 2005;96(8): 1100-6.

9. Yang TH, Park SW, Hong MK, Park DW, Park KM, Kim YH, et al. Impact of diabetes mellitus on angiographic and clinical outcomes in the drug-eluting stents era. Am J Cardiol. 2005;96(10):1389-92.

10. Mulukutla SR, Vlachos HA, Marroquin OC, Selzer F, Holper EM, Abbott JD, et al. Impact of drug-eluting stents among insulin-treated diabetic patients: a report from the National Heart, Lung, and Blood Institute Dynamic Registry. J Am Coll Cardiol Intv. 2008;1(2):139-47.

11. Kirtane AJ, Ellis SG, Dawkins KD, Colombo A, Grube E, Popma JJ, et al. Paclitaxel-eluting coronary stents in patients with diabetes mellitus: pooled analysis from 5 randomized trials. J Am Coll Cardiol. 2008;51(7):708-15. 